\begin{tabular}{|c|c|c|}
\hline & Int.J.Curr.Microbiol.App.Sci (2021) 10(08): 188-193 & \\
\hline & $\begin{array}{l}\text { International Journal of Current Microbiology and Applied Sciences } \\
\text { ISSN: 2319-7706 Volume } 10 \text { Number } 08(\mathbf{2 0 2 1 )} \\
\text { Journal homepage: http://www.ijcmas.com }\end{array}$ & 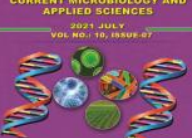 \\
\hline $\begin{array}{l}\text { EXCELLENT } \\
\text { PUBLISHERS }\end{array}$ & & wwwwijemas.com \\
\hline
\end{tabular}

\title{
Ultrasonographic Ocular Biometry for the Diagnosis of Ophthalmic Disorders in Dogs
}

\author{
B. Lavanya*, Syam K. Venugopal, K. D. John Martin, \\ Soumya Ramankutty and A. R. Sreeranjini \\ Department of Veterinary Surgery and Radiology, College of Veterinary and Animal Sciences, \\ Kerala Veterinary and Animal Sciences University, Mannuthy- 680651, India \\ *Corresponding author
}

A B S T R A C T

\section{Keywords}

B-mode ocular ultrasonography, posterior segment, dog, ocular biometry

Article Info

\section{Accepted:}

12 July 2021

Available Online:

10 August 2021
The study was aimed to perform B-mode ocular ultrasonography in the dogs to evaluate intraocular structures. 75 dogs irrespective of the age, breed and sex that were presented during the period of August 2019 to December 2020 to University Veterinary Hospitals at Mannuthy and Kokkalai. Among the 75 dogs, 55 dogs were presented with various ocular affections and 20 dogs with clinically normal eyes subjected to the comparative study. Of the 55 dogs, biometry for various ocular parameters were recorded in 50 dogs. All the dogs were subjected to routine clinical and ophthalmic examinations. B-mode scan was performed with manual restraint. Corneal desensitization was achieved by using topical anesthetic, $0.5 \%$ proparacaine hydrochloride. Biometry for ocular parameters like axial length of the eye (D1), vitreous chamber depth (D2), lens diameter (D3) and lens depth (D4) were recorded in all the dogs which differed significantly within the same group and between groups and between normal eyes and affected eyes. Various pathological lesions were diagnosed and imaged in rest of the five dogs whose ocular biometry was not included in the current study.

\section{Introduction}

Eye is the most unique organ in the body with respect to its anatomical position and orientation. Vision plays a major role in a dog's life as it affects its ability to engage in high performance, visually orientated activities, such as guiding the blind, police work, obedience tests, racing, and hunting (Miller and Murphy, 1995). Hence it is essential to diagnose any ocular pathologies in dogs as early as possible as it affects the behavior of the animal as well. In conditions where posterior segment of the eye is obscured due to the presence of pathologies in the anterior chamber, B-mode ultrasonography 
would be helpful in imaging the posterior segment of the eye (Feliciano et al., 2013). Diagnostic ocular ultrasound is a twodimensional imaging technique used to determine anatomical standards and pathological alterations, especially when ocular and intraocular opacities prevent the assessment of the posterior segment of the eye (Vali and Razeghi, 2019). It can be performed with 7.5-10 MHz probe (Kumar, 2012).

\section{Materials and Methods}

The study was conducted in 75 dogs irrespective of age, breed and sex that were presented to University Veterinary Hospitals, Mannuthy and Kokkalai. Among the 75 dogs, 55 dogs were presented with various ocular affections where as 20 dogs with no apparent ocular abnormalities were chosen randomly in order to assess the normal intraocular structures and to have a comparative study. All the dogs were apparently healthy. Detailed physical and clinical examination were carried out and rectal temperature, pulse rate (per min), respiratory rate (per min), capillary refill time and colour of the conjunctival mucous membranes were recorded in all the dogs and included in the study.

Blood samples were collected on the day of presentation (day 0) and two weeks after first visit (day 14) for haemtological and serum biochemical evaluation. Detailed ophthalmic examination was performed in all the dogs. All the dogs were subjected to B-mode ultrasonography adopting either transpalpebral or transcorneal techniques. Prior to the scan, corneal desensitization was achieved by instilling $0.5 \%$ proparacaine hydrochloride topically over the cornea twice with five minutes interval.

In the current study, immersion technique of transcorneal method was followed where the probe was placed in the thumb compartment of examination glove which was filled with copious amount of coupling gel. This was directly placed over the desensitized cornea to obtain the images of intraocular structures. This set up functioned as a standoff pad to obtain clear images of the anterior structures, reduce surface artifacts and minimal contamination to the cornea.

Scan was performed with dogs in sternal recumbency or sitting with manual restraint. Eye lids were held open manually while transcorneal technique was adopted. Scans were performed using MY LAB ${ }^{\mathrm{TM}} 70$ VETXV (e-saote), SpA, Italy with a micro convex probe. Gain was set constant to maintain the uniformity of the images.

Ocular parameters recorded includes axial length (D1) which was the distance between the echoes of the posterior face of the corneal surface and the retina; Vitreous chamber depth (D2) which was the distance between echoes of the posterior capsule of the lens and the retina; Lens diameter (D3) was the distance between echoes of the opposite points of lens equator i.e. lateral and medial zonules of the ciliary body and lens depth (D4) was the distance between echoes of the anterior and posterior capsules of the lens (Fig.1).

Any changes in the echogenicity of the otherwise anechoic aqueous and vitreous chambers were recorded. Ocular parameters were recorded in the horizontal plane to have a wider diameter of the eye to visualize all the intraocular structures.

In five animals which had ocular pathologies affecting the shape of the globe and ocular structures biometry was not recorded but various associated pathological lesions were recorded.

The obtained data was analyzed by statistical software SPSS 24.0. 


\section{Results and Discussion}

In the current study $10 \mathrm{MHz}$ microconvex probe was used for performing B-mode ultrasonography in all the dogs. Normal ocular biometry was performed in 20 dogs with clinically normal eyes. Various intraocular structures were visualized and ocular biometry was measured and recorded. Transcorneal technique was performed in 74 dogs where as tranpalpebral technique was performed in one dog which had buphthalmos due to ocular melanoma.

Out of the 55 dogs with various affections, ocular biometry was recorded in 50 dogs. Among the 50 dogs, various affections were diagnosed and recorded like cataract $(\mathrm{n}=27,54$ per cent), glaucoma ( $\mathrm{n}=9,18$ per cent), retinal detachment $(\mathrm{n}=5,10$ per cent), pigmentary keratitis ( $\mathrm{n}=2,4$ per cent), hyphema $(\mathrm{n}=2,4$ per cent), nuclear sclerosis ( $\mathrm{n}=3,6$ per cent) and vitreous abnormalities ( $\mathrm{n}=2,4$ per cent) and dogs were grouped based on the ocular affections. Cataracts were further divided into sub-groups as immature, mature and hypermature based on the clinical and ultrasonographical appearance of the lens.

Ocular biometry for various parameters were recorded in all the dogs in different disease groups and compared with that of the biometrical values of the clinically normal eyes and compared within different cataract groups. The mean \pm SE values for the ocular biometrical values within different disease groups were depicted in the Table. 1

The mean \pm SE values for the ocular biometry of affected eyes and normal eyes were depicted in the Table 2. In the current study, 7.5-10 $\mathrm{MHz}$ microconvex probe for performing the B- mode ultrasonography and it yielded cross sectional images of the intraocular structures as reported by Kumar (2012). Martins et al., (2010) and Silva et al.,
(2010) reported use of $10 \mathrm{MHz}$ and $8.5 \mathrm{MHz}$ probe, respectively for ocular ultrasonography in dogs which yielded clear images of the intraocular structures. Transcorneal technique gave better quality and sharp images of the posterior segment of the eye (Toni et al., 2013 and Labruyere et al., 2007). The immersion technique gave better quality images with improved visualization of the anterior chamber and it functioned as a standoff pad. Sanchez et al., (2017) and Philip et al., (2017) reported that they could obtain clear images using this technique. Images obtained by transpalpebral technique did not yield sharp images which was also reported by Vali and Razeghi (2019).

In the normal eye of dogs three, fluid filled cavities (anterior chamber, posterior chamber and vitreous chamber) were visualized. Anterior and Vitreous chamber were anechoic in appearance (Whitcomb, 2002). The cornea could be visualized as a thin curvilinear hyperechoic line parallel to the probe in all the dogs as reported by Paunksnis et al., (2001). Anterior chamber appeared anechoic which was filled with aqueous humor. Anterior chamber was bordered anteriorly by the cornea, laterally and medially by iris and ciliary body and posteriorly the anterior capsule of the lens. Iris was found in continuation with the globe and ciliary body in the form of a single line. Posterior chamber could be located between the ciliary body and the lens at the posterior aspect. (Whitcomb, 2002).

The lens appeared anechoic in normal eyes with curvilinear anterior and posterior capsules being echogenic. Under the posterior margin of the lens, vitreous body was seen as an anechogenic chamber, filled with vitreous humor (Spaulding, 2008). Vitreous was bordered anteriorly by posterior capsule of the lens and posteriorly by posterior wall of the eye ball. 
Table.1 Biometrical measurements (in $\mathrm{mm}$ ) of different diseased groups

\begin{tabular}{|c|c|c|c|c|c|c|c|}
\hline \multicolumn{2}{|c|}{ Variable } & \multirow{2}{*}{\begin{tabular}{|c|}
$\begin{array}{c}\text { Pigmentary } \\
\text { Keratitis }\end{array}$ \\
18.5 \\
\end{tabular}} & \multirow{2}{*}{$\begin{array}{c}\text { Retinal detachment } \\
19.56 \pm 0.61\end{array}$} & \multirow{2}{*}{$\begin{array}{c}\text { Nuclear } \\
\text { Sclerosis } \\
18.43 \pm 0.33\end{array}$} & \multirow{2}{*}{$\begin{array}{c}\text { Hyphema } \\
19.60 \\
\end{array}$} & \multirow{2}{*}{$\begin{array}{l}\text { Glaucoma } \\
20.1 \pm 0.46\end{array}$} & \multirow{2}{*}{$\begin{array}{c}\begin{array}{c}\text { Vitreous } \\
\text { Abnormalities }\end{array} \\
19.80 \\
\end{array}$} \\
\hline$\stackrel{0}{2}$ & D1 & & & & & & \\
\hline$\stackrel{5}{\leftarrow}$ & D2 & 9.25 & $9.24 \pm 0.50$ & $8.70 \pm 0.59$ & 9.05 & $9.64 \pm 0.67$ & 10.05 \\
\hline .010 & D3 & 12.05 & $11.6 \pm 0.58$ & $11.3 \pm 0.76$ & 11.95 & $12.7 \pm 0.58$ & 10.75 \\
\hline & D4 & 7.35 & $7.02 \pm 0.39$ & $6.37 \pm 0.18$ & 6.65 & $7.93 \pm 0.51$ & 11.05 \\
\hline \multirow{4}{*}{ 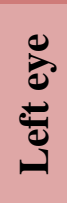 } & D1 & 17.3 & $18.84 \pm 0.18$ & $18.03 \pm 0.92$ & 19.40 & $18.82 \pm 0.39$ & 19.40 \\
\hline & D2 & 8.8 & $8.88 \pm 0.22$ & $10.13 \pm 1.81$ & 9.50 & $9.36 \pm 0.35$ & 8.90 \\
\hline & D3 & 12.35 & $9.78 \pm 1.29$ & $12.17 \pm 0.86$ & 11.70 & $11.07 \pm 0.88$ & 10.90 \\
\hline & D4 & 6.85 & $9.38 \pm 1.62$ & $6.5 \pm 0.15$ & 6.30 & $8.40 \pm 0.98$ & 6.90 \\
\hline
\end{tabular}

Table.2 Comparison of biometry (in $\mathrm{mm}$ ) between diseased group and normal group

\begin{tabular}{|c|c|c|c|c|}
\hline \multirow{3}{*}{ Right eye } & Variable & Diseased & Normal & t-value \\
\cline { 2 - 5 } & Axial length & $19.43 \pm 0.22$ & $16.09 \pm 0.32$ & $8.450^{* *}$ \\
\cline { 2 - 5 } & Vitreous depth & $9.61 \pm 0.25$ & $7.91 \pm 0.14$ & $5.979 * *$ \\
\cline { 2 - 5 } & Lens diameter & $12.08 \pm 0.24$ & $9.52 \pm 0.17$ & $8.756^{* *}$ \\
\cline { 2 - 5 } Left eye & Lens depth & $7.1 \pm 0.28$ & $5.68 \pm 0.14$ & $4.569^{* *}$ \\
\cline { 2 - 5 } & Axial length & $18.89 \pm 0.19$ & $16.24 \pm 0.3$ & $7.587^{* *}$ \\
\cline { 2 - 5 } & Vitreous depth & $9.22 \pm 0.21$ & $7.93 \pm 0.13$ & $5.267 * *$ \\
\cline { 2 - 5 } & Lens diameter & $11.94 \pm 0.29$ & $9.41 \pm 0.17$ & $7.516^{* *}$ \\
\cline { 2 - 5 } & Lens depth & $7.55 \pm 0.29$ & $5.69 \pm 0.15$ & $5.659^{* *}$ \\
\hline
\end{tabular}

** Significant at 0.01 level

Fig.1 Ultrasonogram showing measurement of ocular echo-biometric indices

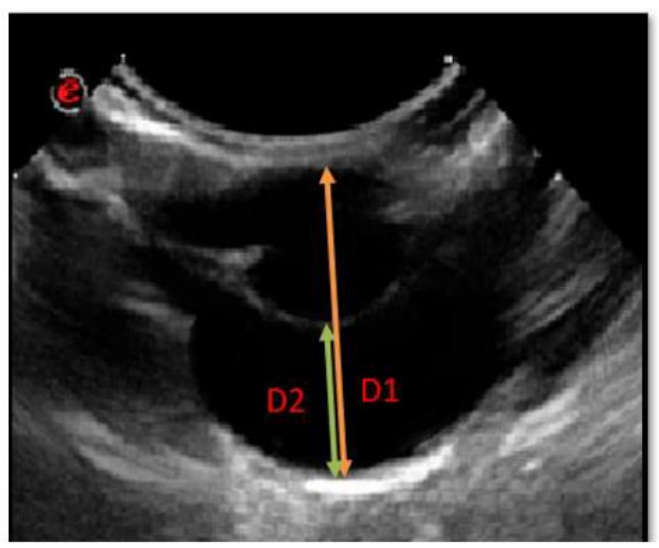

D1- AXIAL LENGTH

D2- VITREOUS

CHAMBER DEPTH

D3- LENS DIAMETER

D4- LENS DEPTH

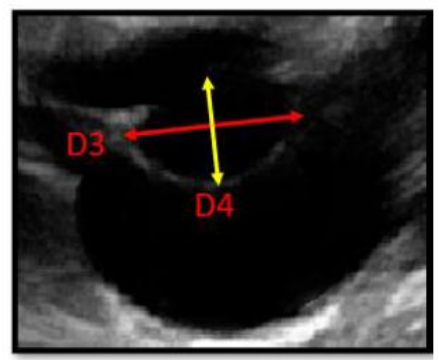

At the posterior wall of the eye ball, the optic disc could be imaged as a thick hyperechoic structure which was evidently more echogenic than adjacent structures (Whitcomb, 2002).
The retina, choroid and sclera were visualized as a single hyperechoic line, which was in contiguous with the iris and could not be visualized separately (Scotty et al., 2004). 
Extending from the optic disc, optic nerve was seen in the form of a hyperechoic cone like structure. Surrounding the optic nerve, retrobulbar fat and ocular muscles could be seen as hypoechoic structures with mottled appearance (Kumar, 2012). In all the cases of cataract, the anterior and posterior capsules of the lens appeared hyperechoic (Kumar, 2012). Nine dogs were diagnosed with glaucoma characterized by increased axial length (Gelatt, 2013). In cases of retinal detachment, the detached retina appeared as "V" shaped hyperechoic structure as reported by Whitcomb (2002). Whitcomb (2002) described this type of retinal detachment as seagull wing appearance.

Two dogs that were presented with hyphema were clinically diagnosed to be suffering from canine monocytic Ehrlichiosis. Similar observations were made by Massa et al., (2002). The ultrasonographic findings revealed no apparent changes in the intraocular changes except for presence of hypoechoic changes in the anterior chamber which was due to the hyphema (Ofri, 2007). In dogs with pigmentary keratitis conventional ophthalmic examination could not be performed due to pigmented and opaque cornea (Anoop et al., 2015). In this group no abnormalities associated with intraocular structures were visualized. Nuclear sclerosis could be identified as a thin hyperechoic rim with in the lens capsule (Whitcomb, 2002). Two vitreous abnormalities like asteroid hyalosis and synchysis scintillans were observed in one case each. The former which was due to accumulation of calciumphospholipid complexes in the vitreous chamber could be seen as mobile with relation to the eye ball as observed by Labrueyre et al., (2007) and Valetini et al., (2010). This was an age related vitreous degeneration as reported by Krishnan et al., (2020). Synchysis scintillans appeared like glistening structures with snow flurry appearance due to presence of cholesterol particles in liquefied vitreous as reported by Boeve and Stades (2007).

There was significant difference in the axial length between the immature, mature and hypermature cataracts in both the eyes. Similar observations were reported by Kumar (2012). There was no significant difference in the rest of the parameters as observed by Silva et al., (2010) and Williams (2004). The biometrical values of the lens observed in this study were similar to the observations made by Ganesan and Iyer (2018). There was significant difference in all the measurements of all the ocular parameters when affected eyes are compared with normal eyes. These findings were in correlation with the observations reported by Kumar (2012).

B-mode ultrasonography was useful in diagnosing various ocular affections. Ocular biometry for various parameters of normal eyes would help us in assessing the changes with respect to that of the diseased eyes. It would be very essential especially while evaluating the intraocular structures when the anterior segment was diseased and opaque.

\section{References}

Anoop, S., Devanand, C. B. and Syam, K. V. 2015. Assessment of grading and isolation of bacterial organisms in canine pigmentary keratitis. International J Advanced Res. 3: 440-443.

Boevé, M. H. and Stades, F. C. 2007. Diseases and surgery of the canine vitreous. Vet. Ophthalmol. 4: 932-943.

Feliciano, M. A. R., Abrahim, M. A., Peixoto, R. V. R., Yasunaga, K. L., Vicente, W. R. R. and Galera, P. D. 2013. Contribution of ocular B-mode and triplex Doppler in the evaluation of 10 Poodle dogs with cataracts.Arq.Bras. Med. Vet. Zootec.65: 359-363.

Ganesan, S. and Iyer, R. C. 2018. Ocular ultrasonographic evaluation of cataractous and pseudophakic eyes in dogs. Turkish J. Vet. Anim. Sci. 42: 611-616. 
Gelatt, K. N. 2013. Essentials of Veterinary Ophthalmology. (5 ${ }^{\text {th }}$ Ed.). John Wiley and Sons, Oxford, 1908p.

Krishnan, H., Diehl, K., Stefanovski, D. and Aguirre, G. D., 2020. Vitreous degeneration and associated ocular abnormalities in the dog. sVet. Ophthalmol. 23: 219-224.

Kumar, D. 2012. B-Mode Ocular Ultrasonography in Dogs and Horses. M.V.Sc. Thesis, Anand Agricultural University, Anand.102p.

Labruyere, J. J., Hartley, C., Rogers, K., Wetherill, G., Mcconnell, J.F. and Dennis, R., 2008. Ultrasonographic evaluation of vitreous degeneration in normal dogs. Vet. Radiol. Ultrasound. 49:165-171.

Martins, B. C., Rodrigues Jr, E. F., Souza, A. L., Almeida, D. E., Brito, F. L., Canola, J.C., Brooks, D. and Laus, J. L. 2010. A and B mode ultrasonography in preoperative evaluation of lens and posterior segment of dogs eyes with cataract. Pesqui. Vet. Bras. 30: 121-126.

Massa, K. L., Gilger, B. C., Miller, T. L. and Davidson, M. G. 2002. Causes of uveitis in dogs: 102 cases (1989-2000). Vet. Ophthalmol. 5: 93-98.

Miller, P. E. and Murphy, C. J. 1995. Vision in dogs. J. Am. Vet. Med. Ass. 207: 12.

Ofri, R. 2007. Blindness in Veterinary ophthalmology: examination, causes and treatment. SCIVAC. $56^{\text {th }}$ International congress.

Paunksnis, A., Svaldeniene, E., Paunksniene, M. and Babrauskiene, V. 2001. Ultrasonographic evaluation of the eye parameters in dogs of different age. Ultragarsas. 39: 48-51.

Philip, L. M., Ramani, C., Williams, B. J. and Ushakumari, S. 2017. Ultrasonographic anatomy and biometry of eye in calves and goats. I. J. Ani. Sci. 87: 175-176.
Sánchez Bustamante, L. M, Rivas Guerrero, J. F and Vargas Pinto, P. A. 2017. Basic ocular ultrasound examination in dogs (real-time Bmode). Rev. Med. Vet. 33: 113-124.

Scotty, N. C., Cutler, T. J., Brooks, D. E. and Ferrell, E. 2004. Diagnostic ultrasonography of equine lens and posterior segment abnormalities. Vet. Ophthalmol. 7: 127-139.

Silva, M. L., Martins, B. C., Ribeiro, A. P., Souza, A. L. and Laus, J. L., 2010. A-and B-modes echobiometry in cataractous and noncataractous eyes of English Cocker Spaniel dogs. Arq. Bras. Med. Vet. Zootec. 62: 1080-1085

Spaulding K. 2008. Eye and orbit. In: Penninck D, Anjou MA, editors. Atlas of small animal ultrasonography (2 $2^{\text {nd }}$ Ed.). Blackwell Publishing. Hoboken, New Jersey, USA. 4990.

Toni, M. C., Meirelles, A. É. W., Laus, J. L. and Canola, J. C. 2013. Ophthalmic ultrasound of dogs with different skull conformations. Rev. Bras. Ciênc.Agr.8: 331-335.

Valentini, S., Tamburro, R., Spadari, A., Vilar, J.M. and Spinella, G. 2010. Ultrasonographic evaluation of equine ocular diseases: a retrospective study of 38 eyes. $J$. Equine Vet. Sci. 30: 150-154.

Vali, R. and Razeghi, M. 2019. Comparison of transcorneal and transpalpebral ultrasonographic measurements of the eye in Iranian Mix Breed Dog. Iranian J. Vet. Sur. 14: 91-96.

Whitcomb, M. B. 2002. How to diagnose ocular abnormalities with ultrasound. AAEP proceedings. 48: 272-275.

Williams, D. L. 2004. Lens morphometry determined by B-mode ultrasonography of the normal and cataractous canine lens. Vet. ophthalmol. 7: 91-95.

\section{How to cite this article:}

Lavanya, B., Syam K. Venugopal, K. D. John Martin, Soumya Ramankutty and Sreeranjini, A. R. 2021. Ultrasonographic Ocular Biometry for the Diagnosis of Ophthalmic Disorders in Dogs. Int.J.Curr.Microbiol.App.Sci. 10(08): 188-193.

doi: https://doi.org/10.20546/ijcmas.2021.1008.023 